\title{
Ketamine normalizes high-gamma power in the anterior cingulate cortex in a rat chronic pain model
}

\author{
Isabel D. Friesner ${ }^{1}$, Erik Martinez ${ }^{1}$, Haocheng Zhou' ${ }^{1}$, Jonathan Douglas Gould ${ }^{3}$, Anna Li' , Zhe Sage Chen 24,5 (D), \\ Qiaosheng Zhang ${ }^{1 *^{*}}$ (D) and Jing Wang ${ }^{1,4,5^{*+}}$ (D)
}

\begin{abstract}
Chronic pain alters cortical and subcortical plasticity, causing enhanced sensory and affective responses to peripheral nociceptive inputs. Previous studies have shown that ketamine had the potential to inhibit abnormally amplified affective responses of single neurons by suppressing hyperactivity in the anterior cingulate cortex (ACC). However, the mechanism of this enduring effect has yet to be understood at the network level. In this study, we recorded local field potentials from the ACC of freely moving rats. Animals were injected with complete Freund's adjuvant (CFA) to induce persistent inflammatory pain. Mechanical stimulations were administered to the hind paw before and after CFA administration. We found a significant increase in the high-gamma band (60-100 Hz) power in response to evoked pain after CFA treatment. Ketamine, however, reduced the high-gamma band power in response to evoked pain in CFA-treated rats. In addition, ketamine had a sustained effect on the high-gamma band power lasting up to five days after a single dose administration. These results demonstrate that ketamine has the potential to alter maladaptive neural responses in the ACC induced by chronic pain.
\end{abstract}

Keywords: Chronic pain, Anterior cingulate cortex, Ketamine, Gamma band power, Local field potential

\section{Introduction}

Chronic pain impacts around $20 \%$ of people globally [1]. Current treatments cause many side effects due to the incomplete understanding of the mechanisms of chronic pain [2]. For example, there has been a dramatic rise in prescription of opioid drugs to treat chronic postoperative pain [3-5], which has in turn lead to sedation, respiratory depression, dependence and even addiction [5, 6]. Therefore, there is an urgent need not only to propel our understanding of the mechanism of chronic pain, but

\footnotetext{
*Correspondence: qiaosheng.zhang@nyulangone.org; jing. wang2@nyulangone.org

${ }^{\dagger}$ Qiaosheng Zhang and Jing Wang contributed equally to this work ${ }^{1}$ Department of Anesthesiology, Perioperative Care and Pain, New York University School of Medicine, New York, NY 10016, USA

Full list of author information is available at the end of the article
}

also to find alternative effective analgesics with minimal side effects [7].

Recently, administrations of low, sub-anesthetic doses of ketamine either alone or as an adjunct to opioid therapies have been shown to be an effective treatment in postoperative and acute pain settings [8-12]. In addition, ketamine has long been safely used as an general anesthetic and analgesic agent [13]. Recently, the FDA approved a new intranasal preparation of ketamine for the treatment of acute depressive symptoms [14]. Specifically regarding chronic pain states, human and animal studies have shown the effectiveness of ketamine injections in eliciting a rapid response to peripheral nociceptive inputs, and in some cases, showing sustained analgesic efficacy lasting up to a week [15-18]. These fact-acting and persistent effects of ketamine prompt further investigation into its analgesic mechanisms.

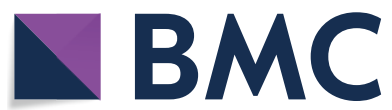

c The Author(s) 2020. This article is licensed under a Creative Commons Attribution 4.0 International License, which permits use, sharing, adaptation, distribution and reproduction in any medium or format, as long as you give appropriate credit to the original author(s) and the source, provide a link to the Creative Commons licence, and indicate if changes were made. The images or other third party material in this article are included in the article's Creative Commons licence, unless indicated otherwise in a credit line to the material. If material is not included in the article's Creative Commons licence and your intended use is not permitted by statutory regulation or exceeds the permitted use, you will need to obtain permission directly from the copyright holder. To view a copy of this licence, visit http://creativeco mmons.org/licenses/by/4.0/. The Creative Commons Public Domain Dedication waiver (http://creativecommons.org/publicdomain/ zero/1.0/) applies to the data made available in this article, unless otherwise stated in a credit line to the data. 
Recent studies have shown chronic pain could impair the balance between sensory and affective components of pain, leading to a generalized, anatomically nonspecific enhancement in the aversive response to acute noxious stimuli [19-21]. In addition, chronic pain alters synapses and circuits in the cerebral cortex and contributes to abnormalities in pain affect [22-24]. The anterior cingulate cortex (ACC) has been known to modulate the affective-motivational component of pain and lead to hyperactivity of neuronal components in response to pain $[3,7,24-26]$. The ACC mediates the aversive response to pain evoked by noxious stimulations. [4, 26-28] These findings have been replicated in other model systems, including rabbits and monkeys, where increases in extracellular activity in the ACC have been observed to occur in response to stimulations in freely moving animals and humans [29-32]. Furthermore, studies have shown that ACC neurons undergo synaptic plasticity in chronic pain conditions $[7,25,33]$. Thus, there is a possibility that neuronal responses to noxious stimuli in the ACC may show distinct patterns in the chronic pain state.

Ketamine has been shown to affect multiple areas of the brain, including the ACC $[34,35]$. Our previous animal study showed that a single dose of ketamine was able to inhibit this abnormal enhancement in pain aversion for 5 days in the ACC [36]. $N$-Methyl-D-aspartate receptors (NMDARs) are glutamate receptors that play a role in the excitatory response of the central nervous system. [37, 38] In a chronic pain state, changes in neuroplasticity lead to excessive activation of NMDARs [39, 40]. As a NMDARs antagonist, ketamine likely influences the affective-motivational component through regulating neuroplasticity $[41,42]$. However, the mechanism of this enduring effect has yet to be widely understood since glutamate is a broadly distributed neurotransmitter in the brain. Recently, we observed a reduction in the hyperactivity of ACC neurons in chronic pain states after ketamine administration, allowing individual neuron to return their spiking rate in response to acute noxious stimuli back to baseline levels [36].

In contrast to action potentials (or spikes), local field potentials (LFPs) measure collective behavior of ensemble neurons, and frequency-specific LFPs are thought to process distinct network information. For instance, the high-gamma $(60-100 \mathrm{~Hz})$ oscillatory activity is often seen to correlate with spike synchrony and could be utilized as a substitute for assessing output of neuronal activity. Moreover, neural oscillations in broadband power spectrum have been shown to be involved in neuropathic pain, characteristics of which can be utilized to analyze aspects of pain and pain relief [43]. Thus, we hypothesize that ketamine can rescue or reverse enhanced high-gamma band power in the ACC in response to noxious stimuli in the chronic pain condition.

In this study, we recorded LFPs in the ACC in freely moving rats from a classic chronic pain model-complete Freund's adjuvant (CFA) model of inflammatory pain. Mechanical pinprick was used to generate nociceptive inputs, and LFP spectral powers were evaluated before and after ketamine treatment. Interestingly, we found chronic pain increased the high-gamma band power in ACC neurons. A single sub-threshold anesthetic dose of ketamine, however, reversed high-gamma enhancement in response to evoked pain in the chronic pain condition. Therefore, our results support that ketamine could rescue chronic pain induced neuroplasticity at a network level.

\section{Results}

\section{Elevation in the high-gamma band power in the ACC in response to chronic pain}

Extracellular activities (spikes and LFPs) were recorded from chronically implanted tetrodes in the ACC region of awake, freely moving rats (Fig. 1a, b). Multi-channel LFP data were collected over multiple sessions, each consisting of continuous recordings over at least 30 noxious pinprick stimulations to the rat's hind paw, contralateral to the ACC tetrode implants, though a mesh table (Fig. 1a). Single-trial LFP raw data were collected and averaged to produce trial-averaged LFP raw traces, as single-trial neural signals can be very noisy. The trial-averaged LFP raw data were then used to generate spectrograms, and we analyzed the broadband power spectra $(4-100 \mathrm{~Hz}$, "all frequency" with the exception of low-frequency $(1-3 \mathrm{~Hz})$ bands, which often contain artifacts). In the pre-CFA state, there was minimal activity in the gamma band as seen in the trial-averaged LFP spectrogram (Fig. 2a).

Next, we induced chronic pain by injecting complete Freund's adjuvant (CFA) to the rat's hind paw, ipsilateral to the ACC recording sites [44]. In contrast to rats in the naïve state, CFA-injected rats demonstrated a noticeable increase in the activity in the high-gamma region as shown in the trial-averaged LFP spectrogram (Fig. 2b). Furthermore, as demonstrated by the trial-averaged LFP raw traces, the peak in the chronic pain state is greater than the peak in the naïve state (Fig. 2a, b). We quantitated the LFP power in the naïve and chronic pain conditions, and compared power in the theta $(4-8 \mathrm{~Hz})$, beta (8-15 Hz), alpha (15-30 Hz), low-gamma $(30-60 \mathrm{~Hz})$, and high-gamma $(60-100 \mathrm{~Hz})$ bands. There was no statistical significance between the naïve and chronic pain conditions in the theta, alpha, beta, and low-gamma bands (Fig. 2c-g). However, there was a statistically significant difference in the high-gamma band, demonstrating an increase in power in the high-gamma band in CFA-treated rats $(p=0.0084$, Paired t-test, Fig. $2 h)$. The 


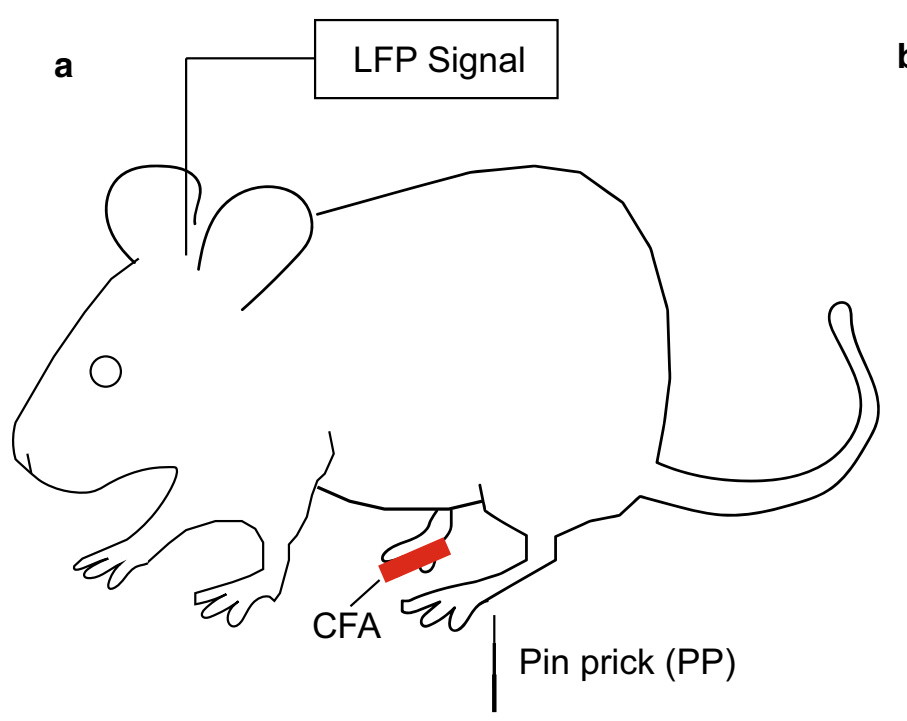

b

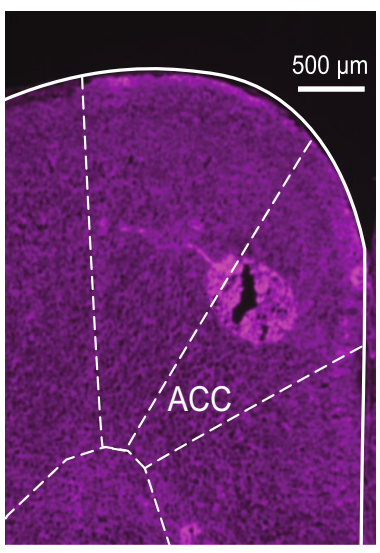

Fig. 1 Experimental Design. a Pinprick stimulations were conducted in the uninjured paw contralateral to the CFA injection. Local field potentials (LFPs) were recorded from the anterior cingulate cortex (ACC) contralateral to peripheral stimulations. $\mathbf{b}$ Location of recording electrodes in the ACC

data indicates that chronic pain induces increased LFP activity and power in the high-gamma band.

\section{A single low dose of ketamine reduces the LFP power in the ACC}

CFA-treated rats were administered two days later, intraperitoneally, with either saline (control) or ketamine (Fig. 3a, b). A sub-anesthetic dose of ketamine (10 $\mathrm{mg} \mathrm{kg}^{-1}$ ) was chosen, based on previous studies [45-47]. The saline-treated rats demonstrated a sustained increase in power in the high-gamma region, as seen in the trial-averaged LFP spectrogram (Fig. 3c). In contrast, ketamine-treated rats showed a decrease in activity in the high-gamma band, as seen in the trialaveraged LFP spectrogram (Fig. 3d). Similar to rats in the pre-CFA state, the trial-averaged LFP raw trace for the control group, saline-treated rats, showed a larger peak compared with the trial-averaged LFP raw trace for ketamine-treated rats (Fig. 3c, d). We also examined the power in other frequency bands, and found no statistical significance in the theta or alpha frequency bands (Fig. 3e, f). We did observe, however, a statistically significant decrease in power in the beta and low-gamma bands $(p=0.0229$ and $p=0.0267$, respectively, unpaired t-test, Fig. 3g, h). The power reduction in the high-gamma band was substantial, as the power returned to the baseline value prior to CFA injection $(\mathrm{p}=0.0086$, unpaired $\mathrm{t}$-test, Fig. 3i). These data indicate that ketamine has the potential to reduce the power in the beta and low-gamma bands in CFA-treated rats. Specifically in the high-gamma band, ketamine appears to inhibit the increase of power associated with chronic pain.

\section{Ketamine provides sustained inhibition of LFPs in the high-gamma band}

Previous research suggests ketamine produces antidepressant effects that can last multiple days after a single administration $[13,15,48-50]$. To test whether a single injection of ketamine could reduce ACC activity over a sustained period of time, we measured LFPs 5 days after the ketamine injection (Fig. 4a). Five days after the ketamine injection, the trial-averaged spectrogram demonstrates a sustained power decrease in the highgamma band (Fig. 4b). Furthermore, the peak of the trial-averaged LFP raw trace was consistent with the peaks of pre-CFA and day two ketamine-treated traces (Fig. 4b). Furthermore, we analyzed LFP power quantitatively and found a statistically significant difference comparing Day 2 and Day 5 after ketamine injection to the pre ketamine chronic pain state $(\mathrm{p}=0.0140$ and $\mathrm{p}=0.0322$, respectively, One-way ANOVA using Dunnett's multiple comparisons, Fig. 4c). These data indicated that a single dose of ketamine is able to inhibit an increase in power in the high-gamma band and restore CFA-treated rats to a pre-CFA level of activity for up to five days. Thus, for rats in the chronic pain state, LFPs in the ACC demonstrate a sustained reduction in population activity due to a single ketamine injection. 


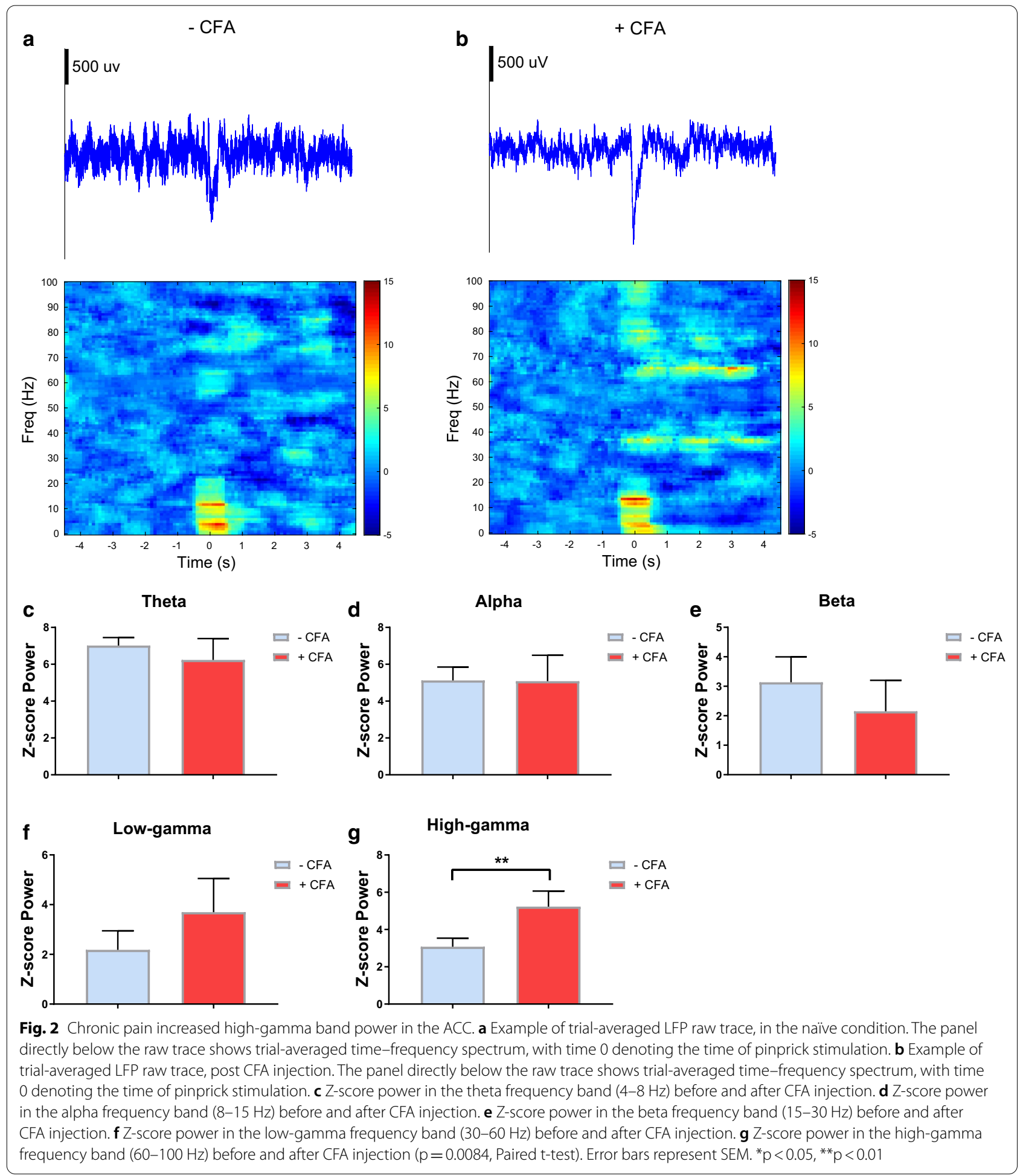

\section{Discussion}

This study is aimed at investigating LFP responses in the ACC to noxious stimulations in the chronic pain state and the effect of ketamine on these responses. We have demonstrated that chronic inflammatory pain causes an increase in spectral power in the high-gamma band in the $\mathrm{ACC}$, and that ketamine reduces this abnormal increase in high-gamma power for at least 5 days. 


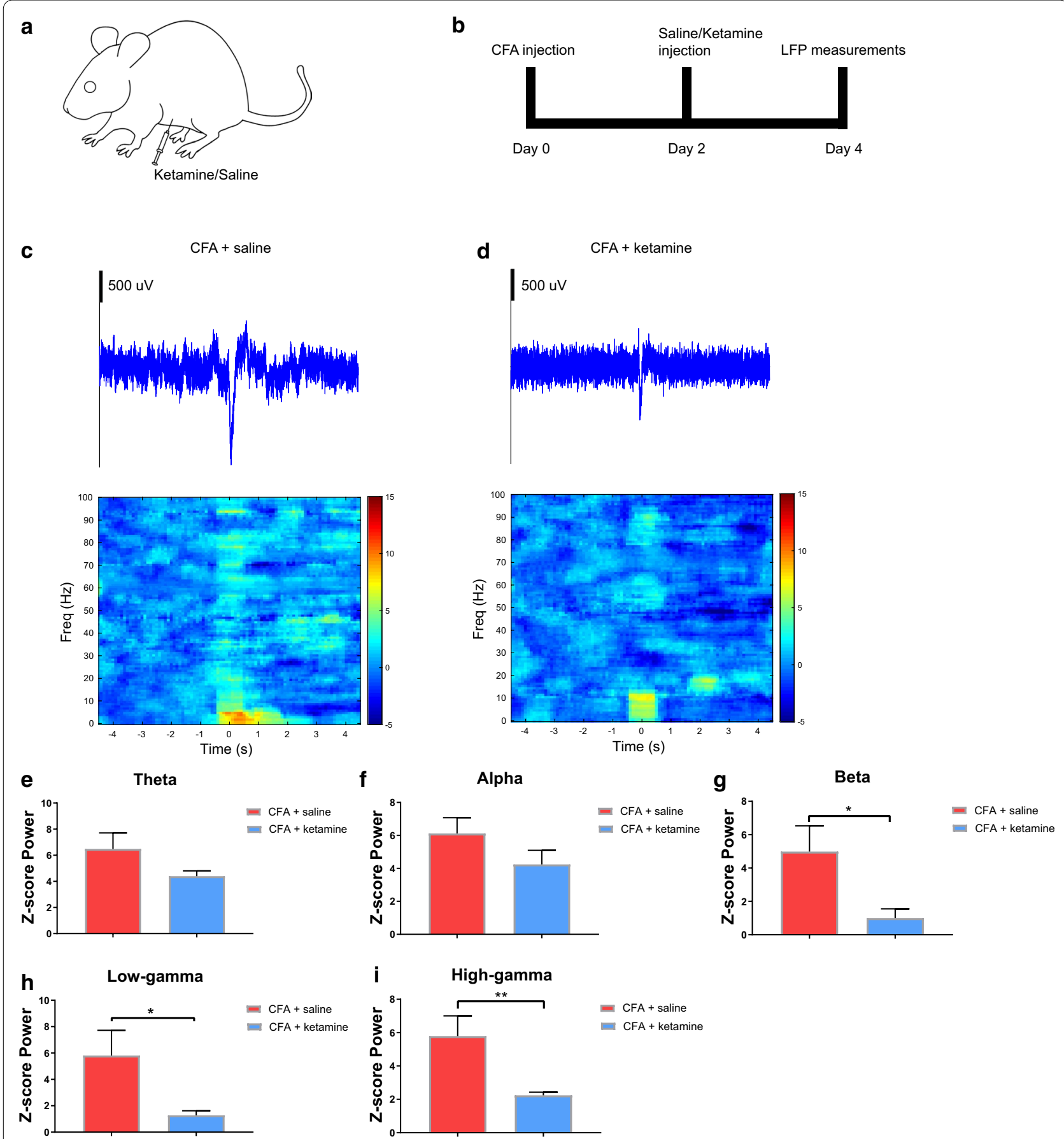

Fig. 3 Ketamine inhibited the enhancement of high-gamma band power in CFA-treated rats. a Rats received either a ketamine or saline intraperitoneal injection. b Timeline for CFA, saline, and ketamine injections. c Example of trial-averaged LFP raw trace, in the chronic pain condition two days after saline injection. The panel directly below the raw trace shows trial-averaged time-frequency spectrum, with time 0 denoting the time of pinprick stimulation. $\mathbf{d}$ Example of trial-averaged LFP raw trace, in the chronic pain condition two days after ketamine injection. The panel directly below the raw trace shows trial-averaged time-frequency spectrum, with time 0 denoting the time of pinprick stimulation. e Z-score power in the theta frequency band $(4-8 \mathrm{~Hz})$ two days after saline or ketamine injection. $\mathbf{f} Z$-score power in the alpha frequency band $(8-15 \mathrm{~Hz})$ two days after saline or ketamine injection. $\mathbf{g}$ Z-score power in the beta frequency band $(15-30 \mathrm{~Hz})$ two days after saline or ketamine injection $(p=0.0229$, unpaired t-test). $\mathbf{h}$ Z-score power in the low-gamma frequency band $(30-60 \mathrm{~Hz})$ two days after saline or ketamine injection $(p=0.0267$, unpaired t-test). $\mathbf{i}$ Z-score power in the high-gamma frequency band $(60-100 \mathrm{~Hz})$ two days after saline or ketamine injection $(p=0.0086$, unpaired t-test). Error bars represent SEM. ${ }^{*} p<0.05 ;{ }^{* *} p<0.01$ 


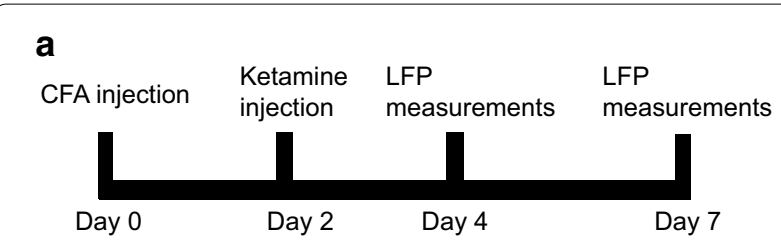

b CFA + ketamine (Day 5)

$500 \mathrm{uV}$
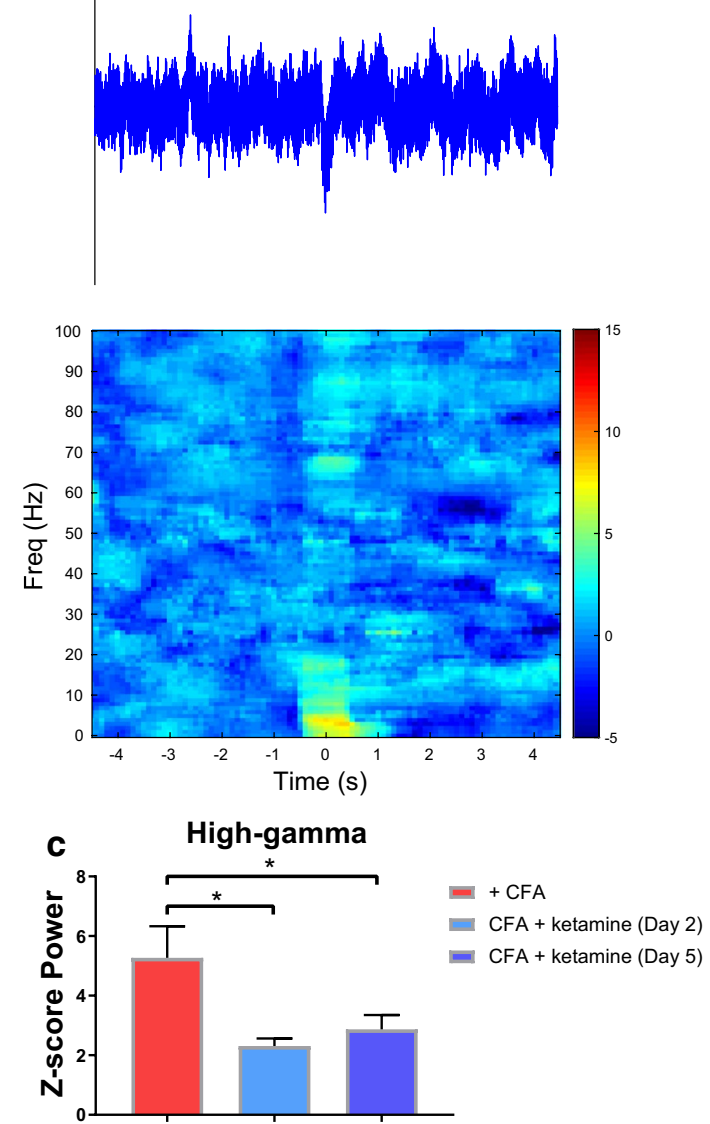

Fig. 4 Ketamine had a long term effect of inhibition on the high-gamma band power in the ACC in chronic pain state. a Timeline for CFA and ketamine injections. $\mathbf{b}$ Example of trial-averaged LFP raw trace, in the chronic pain condition five days after ketamine injection. The panel directly below the raw trace shows trial-averaged time-frequency spectrum, with time 0 denoting the time of pinprick stimulation. c Z-score power in the high-gamma frequency band $(60-100 \mathrm{~Hz})$ post CFA injection, two days after ketamine injection, and five days after ketamine injection $(p=0.0140$ and $p=0.0322$, respectively, One-way ANOVA with Dunnett's multiple comparisons test). Error bars represent SEM. ${ }^{*} p<0.05$

Changes in gamma oscillations in acute and chronic pain states have been studied in both animal models and human subjects, and our observed increase in high-gamma band power is consistent with previous results [51-56]. Our finding that chronic pain caused increased power in the high-gamma band specifically in the $\mathrm{ACC}$ is consistent with another previous thermal pain study [57]. Interestingly, however, in the current study, we did not observe power enhancement in the theta band, in contrast to what was found with earlier studies in thermal stimulation [57, 58]. One reason for this discrepancy may be that mechanical pinprick stimulations, as opposed to thermal stimulations, elicit a greater theta band power response, thereby occluding further changes in the chronic pain conditions. This hypothesis is indirectly supported by a previous thermal pain study, which demonstrated a smaller change in CFA-induced theta band power elicited by high-intensity noxious stimuli compared with low-intensity noxious stimuli [57]. Importantly, different peripheral sensory neurons may respond to different intensities of mechanical and thermal stimulations [59]. A-delta fibers conduct quickly in response to highly noxious stimuli, particularly highly noxious mechanical stimuli [60]. In contrast, while C-fibers respond to both mechanical and thermal stimuli, they conduct slowly and play a greater role in distinguishing slower changes in temperature and resulting thermal pain intensity [61, 62]. At the molecular level, different mechanosensory or thermal receptors on these pain fibers also respond with different kinetic and thermodynamic profiles $[63,64]$. Thus, changes in cortical oscillations may also reflect changes in peripheral, spinal and thalamic nociceptive pathways, which are complex and diverse in nature.

The role of ACC in pain processing, particularly the processing of affective component of pain, has been well documented [25, 31, 65-71]. Our results here further support these roles. Meanwhile, in a previous study, rats in the chronic pain state that received a ketamine injection demonstrated decreased pain aversion compared to control rats [36]. This study further demonstrated that ketamine likely reduced the affective component of pain by suppressing hyperactivity of ACC neurons in the chronic pain state [36]. In another study, ketamine has been shown to reduce depression-like behaviors in the chronic neuropathic pain state as well [46]. While our current results do not directly link high-gamma changes in the ACC with the analgesic properties of ketamine, in the context of these previous results they lend additional support to the role of ketamine in shaping functional connectivity involving ACC neurons. Future experiments that directly link LFP changes with behavioral observations would further our understanding of the impact of ketamine on the ACC in chronic pain states.

An important pathological feature of chronic pain is an amplified aversive response to noxious stimuli in an 
anatomically nonspecific manner, as found in conditions of fibromyalgia and persistent postoperative pain [19, 21, 72, 73]. Previous animal studies have shown chronic pain can alter synapses and circuits in the cerebral cortex, especially in the ACC $[67,70]$. In a previous study analyzing the effect of ketamine on neuronal firing activity, ACC neuronal firing rates increased in the chronic pain state and returned to naïve levels after a single sub-anesthetic dose of ketamine [36]. Interestingly, in the present study we found that ketamine could reduce the ACC highgamma band power enhancement induced by chronic pain. The time scale of gamma waves $(10-20 \mathrm{~ms})$ corresponds to the temporal window of spike timing-dependent plasticity involved in shaping synaptic connections $[74,75]$. Thus, the high-gamma band power inhibition observed in the current study indicates that ketamine has the potential to reverse abnormal neuronal plasticity developed in the chronic pain state. Furthermore, the power reduction in the high-gamma band in the ACC lasted five days, which is consistent with the known time scale for the effect of ketamine on mood and pain [15-18, 46]. Previous studies have shown that inhibition of the $\mathrm{N}$-methyl-D-aspartate receptors (NMDARs) by ketamine can increase brain derived neurotrophic factor (BDNF) expression in the hippocampus and prefrontal cortex, important regions for pain and mood regulation $[18,76]$. In addition, ketamine can also upregulate mTORC1, a translational regulator, to promote the expression of specific synaptic proteins in the cortex $[45,77,78]$. Through this mechanism, ketamine can cause persistent increase in $\alpha$-amino-3-hydroxy-5-methyl-4-isoxazolepropionic acid (AMPA) receptor-mediated neurotransmission to produce sustained antidepressant effects [79-84]. Thus, at the molecular level, its impact on central glutamate signaling underlies the long-lasting effects of ketamine on pain behaviors and may also mediate its effect on highgamma oscillations in the ACC. Previous studies have also found that ketamine can decrease the power in lowfrequency bands [85-88], and changes in the low-gamma and beta region have in fact been associated with altered glutamatergic levels in the ACC [89]. Interestingly, we also observed decreases in the power of low-gamma and beta bands after ketamine administration in the current study. Thus, ketamine has the potential to alter functional connectivity in the brain through widespread changes in the power spectra.

In our study, ketamine produced anti-aversive effects through actions on the ACC. However, as an antagonist of NMDARs, ketamine is likely to modulate brain state and change neuronal plasticity in many areas, since NMDARs play a critical role in synaptic plasticity throughout cortical and subcortical areas [90-95]. For example, a recent animal study has shown that ketamine can relieve symptoms of depression by blocking bursting in the lateral habenula [49]. In addition, ketamine is able to produce rapid antidepressant responses by inducing prefrontal cortex synaptogenesis and reversing the synaptic deficits caused by chronic stress [96]. A recent study has shown that ketamine is capable of activating GABAergic neurons in the central amygdala in both acute and chronic pain states [97]. Other regions such as the insular cortex, periaqueductal gray, and nucleus accumbens may also be involved in the anti-aversive effects of ketamine. Thus, future studies should focus on examining LFPs from multiple regions to obtain a comprehensive overview of the anti-aversive effects of ketamine at a broader pain network level.

Movement artifacts constitute a technical challenge for LFP recordings in freely moving animals. Multiple steps were taken in the present study to minimize movement artifacts. First, we removed all trials with abnormally high amplitudes, most likely resulting from unrelated or sudden reflexive motion. Second, we verified each trial with a video recording and removed the trial if unrelated movement was observed. Lastly, if a majority of channels contained noisy signal the trial was excluded, and if a majority of all trials in a given session were noisy, the entire session was excluded. In terms of final data analysis, noisy signals as the result of movement are expected to demonstrate an increase in power. Since our data showed a decrease in high gamma band power in the chronic pain state, noisy trials as the result of movement artifact likely did not significantly impact our findings. Nevertheless, to further minimize movement artifacts, future LFP recordings can be done with simultaneous neck electromyography recordings and motion monitoring with an ultra high-speed camera.

Other areas of limitation of this study include the use of a single dose of ketamine and a single chronic pain model. In addition, this study primarily addresses neural changes in response to evoked pain. Chronic pain, however, also includes spontaneously occurring pain episodes. Thus, future studies examining different dosages of ketamine, in the context of multiple pain behaviors, including spontaneous pain behaviors, in additional pain models are needed to further elucidate the cortical mechanisms of ketamine in treating chronic pain.

LFPs bear resemblance to electroencephalogram (EEG) signals, allowing studies of LFPs in animals to be translated to studies of EEG signals in humans [98]. This is supported by findings of increased gamma oscillations and quantification of pain perception in the somatosensory cortex in response to noxious stimuli $[99,100]$. Recent technical development allows a single EEG electrode to record temporal-spectral neural patterns over single-trial stimulations and provide information about 
neuronal responses and sensitivity to pain [101]. Thus, if high frequencies of LFPs can be used as proxies for assessing the neuronal output, our system of inquiry can be potentially translated to EEG signals to introduce a noninvasive method to measure extracellular activity and objectively record and analyze human response to chronic pain.

In conclusion, we found that chronic pain increased power in the high-gamma band in the ACC of rats. A single sub-anesthetic dose of ketamine was able to rescue activity in the high-gamma band to reverse the changes induced by CFA. Furthermore, these effect on highgamma band power lasted up to 5 days after ketamine injection. These findings suggest ketamine can impact network neuronal activity and cortical plasticity in the chronic pain state.

\section{Methods and materials}

\section{Experimental animals}

All procedures in this study were approved by the New York University School of Medicine (NYUSOM) Institutional Animal Care and Use Committee (IACUC) as consistent with the National Institute of Health (NIH) Guide for the Care and Use of Laboratory Animals to ensure minimal animal use and discomfort. Animals consisted of male Sprague-Dawley rats, 250 to 300 g each upon arrival, purchased from Taconic Farms (Albany, NY). Animals were housed at the Mispro Biotech Services Facility in the Alexandria Center for Life Science in a controlled environment, monitoring temperature, humidity, and 12 h (6:30 A.M. to 6:30 P.M.) light-dark cycle. Food and water were available ad libitum. Animals were given on average 14 days to adjust to the new environment before beginning experiments.

\section{Drugs}

Rats were injected with $0.06 \mathrm{~mL}$ of CFA (Mycobacterium tuberculosis, Sigma-Aldrich) to induce inflammatory pain in the injected paw and initiate a chronic pain model. CFA was initially suspended in an oil-saline (1:1) emulsion and subsequently injected subcutaneously into the plantar aspect of the hind paw, ipsilateral to location of recording tetrodes and opposite the paw receiving pinprick stimulations. Ketamine-treated rats received one $0.5 \mathrm{~mL} 10 \mathrm{mg} \mathrm{kg}^{-1}$ injection of ketamine hydrochloride (Ketaset), purchased from Zoetis, intraperitoneally. The control group received an equal volume of saline injected intraperitoneally.

\section{Electrode implant and surgery}

Two twisted $12.7 \mu \mathrm{m}$ polyimide-coated microwires (Sandvik) were used to construct the stereotrodes. The stereotrodes were then mounted in a VersaDrive8
(Neuralynx), similar to other experiments, and dental cement was used to secure the drive to the skull screws $[70,102]$. To reduce electrode impedances to $100-500$ $\mathrm{k} \Omega$, electrode tips were plated with gold. In order to implant stereotrodes, the skull was exposed allowing a craniotomy to be performed over unilateral anterior cingulate cortex (AP + 2.5-3.5 mm, ML 0.8-1.8 mm). Isoflurane $(1.5-2 \%)$ was used to anesthetize rats during implantation. With the tip angled $10^{\circ}$ toward the midline, the electrode bundle was lowered at DV $1.6 \mathrm{~mm}$. The average recovery time post-surgery, before neural recordings, was 1 week.

\section{In vivo electrophysiological recordings}

Rats with electrode implants were place in a recording chamber over a mesh table and given $30 \mathrm{~min}$ prior to the onset of stimulations to adjust to the environment [70]. After the initial adjustment period, approximately 30 trials with variable inter-trial intervals (approximately $1 \mathrm{~min}$ ) were conducted per session in free-moving rats. Inter-trial intervals were set between trials to avoid sensitization. Each trial consisted of a noxious simulation by pricking using a 27 -gauge needle administered to the plantar surface of the hind paw contralateral to location of electrode implant and concluded by paw withdrawals. Throughout all sessions, no physical damage or behavioral sensitization to the paw was discerned. All sessions were recorded with a $120 \mathrm{fps}$ video camera (DMK23U, image source).

\section{Neural data collection and preprocessing}

Stereotrodes were lowered $60 \mu \mathrm{m}$ interval each day before recording. During sessions, neural activity and signals before, during, and after, pinprick stimulations were recorded at a sample rate of $40 \mathrm{kHz}$ using acquisition equipment (OmniPlex D with Digital Headstage Processor, Plexon). Raw data of LFPs was digitally filtered with a bandpass filter between 0.3 and $300 \mathrm{~Hz}$ and then down-sampled to $1 \mathrm{kHz}$.

\section{Histocytochemistry}

Isoflurane was used to anesthetize rats whom were then transcardially perfused with ice-cold phosphate-buffered saline (PBS) and paraformaldehyde (PFA). Brains were fixed in PFA overnight and subsequently transferred for 3 days to $30 \%$ sucrose in PBS to equilibrate [103]. Microm HM252 Cryostat (Thermo Fisher Scientific) was used to collect $20 \mu \mathrm{m}$ coronal sections. These sections were then washed in PBS and covered with a Vectashield mounting medium. If the section contained an electrode it was stained with cresyl violet or hematoxylin and eosin stain and analyzed with a Nikon eclipse 80i microscope with 
a DS-U2 camera head. Animals with incorrect electrode placement were excluded.

\section{Data preprocessing}

Multi-channel LFP signals were saved from Plexon system, and we preprocessed the raw data to remove noisy trials. If sessions had been spike sorted, three channels were chosen based on those that had the greatest signal to noise ratio. If sessions had not been sorted, all channels with available LFP data were used. These denoised single-channel LFP signals were then utilized for subsequent spectrum analyses.

Multiple steps were taken in the present study to minimize movement artifacts. First, we removed all trials with abnormally high amplitudes, most likely resulting from unrelated or sudden reflexive motion. Second, we verified each trial with a video recording and removed the trial if unrelated movement was observed. Lastly, if a majority of channels contained noisy signal the trial was excluded, and if a majority of all trials in a given session were noisy, the entire session was excluded.

\section{Spectrum analysis}

We opted for a multitaper method to compute spectrum analysis of LFP signals. The multitaper method is a spectral analysis technique to reduce bias/variance of spectral estimates [104]. It does this by pre-multiplying the data with orthogonal tapers, known as Slepian functions. We set a half-bandwidth parameter W, which defines the dimensions of the moving window to be $[-\mathrm{W}, \mathrm{W}]$. In order to ensure the Slepian taper functions were contained in frequency and have bias reducing characteristic, W was set to be greater than $1 / \mathrm{T}$, where $\mathrm{T}$ indicates the temporal duration. The multitaper method and spectrum analysis was computationally generated with the Chronux toolbox [105]. The Chronux toolbox is an open source data analysis software (https://chronux.org). Spectrograms were generated using the function 'mtspecgramc.' The parameters for mtspecgramc was [TW $\mathrm{K}]$, where $\mathrm{TW}=3$ and $\mathrm{K}=2 \times \mathrm{TW}-1=5$. TW is the time-bandwidth product and $\mathrm{K}$ is the number of tapers. A single vector was produced by summing all power values in each frequency band (theta 4-8 Hz, alpha 8-15 Hz, beta $15-30 \mathrm{~Hz}$, lowgamma 30-60 Hz, high-gamma $60-100 \mathrm{~Hz}$ ). Then for each frequency band we computed the Z-score of power related to the baseline period $[-4.5,-0.5] \mathrm{s}$ before pinprick simulation, and computed the average over the stimulus range [ $-0.51 .0]$, where 0 denotes the time of pinprick stimulation, to produce a single power value. Estimated power values were then averaged over all selected channels to produce one estimated power value for the given session for a single rat. If the Z-score was positive, it demonstrated an increase in power at the specific frequency band(s).

\section{Statistical analysis}

To compare Z-score power values of rats before CFA injection with rats after CFA injection, we used a Paired t-test. To compare saline injections with ketamine administrations in CFA-treated rats, we used an unpaired t-test. To compare ketamine administration over multiple days in CFA-treated rats, we used a One-way ANOVA test with post-hoc Dunnett's multiple comparisons. We reported the mean \pm SEM statistics in LFP power. For all tests, a $p$ value $<0.05$ was considered statistically significant. All data was analyzed using the GraphPad Prism Version 7 software (GraphPad) and MATLAB (MathWorks).

\section{Abbreviations}

CFA: Complete Freund's adjuvant; LFPs: local field potentials; ACC: anterior cingulate cortex; NMDARs: N-methyl-D-aspartate receptors; EEG: electroencephalogram; AMPA: a-amino-3-hydroxy-5-methyl-4-isoxazolepropionic acid.

\section{Authors' contributions}

QZ and JW designed the project. QZ performed the electrode constructions and implantations. QZ, EM, HZ, performed recordings, IF, QZ and JD performed data analysis. EM and AL performed the Immunohistochemistry. IF, QZ, JW and $\mathrm{ZC}$ wrote the paper. All authors read and approved the final manuscript.

\section{Funding}

This work was supported by the NIH Grant GM115384.

\section{Availability of data and materials}

All the data and code are available from the corresponding author on reasonable request.

\section{Ethics approval and consent to participation}

All animal care and experimental procedures of this study were approved by the New York University School of Medicine (NYUSOM) Institutional Animal Care and Use Committee (IACUC) as consistent with the National Institute of Health $(\mathrm{NIH})$ Guide for the Care and Use of Laboratory Animals to ensure minimal animal use and discomfort.

\section{Consent for publication}

All authors agreed to its submission to the Molecular Brain and, if accepted, to its publication in this journal.

\section{Competing interests}

The authors declare no competing interests.

\section{Author details}

${ }^{1}$ Department of Anesthesiology, Perioperative Care and Pain, New York University School of Medicine, New York, NY 10016, USA. ${ }^{2}$ Department of Psychiatry, New York University School of Medicine, New York, NY 10016, USA. ${ }^{3}$ College of Arts and Sciences, New York University, New York, NY 10003, USA. ${ }^{4}$ Department of Neuroscience \& Physiology, New York University School of Medicine, New York, NY 10016, USA. ${ }^{5}$ Neuroscience Institute, New York University School of Medicine, New York, NY 10016, USA.

Received: 8 July 2020 Accepted: 14 September 2020

Published online: 23 September 2020 


\section{References}

1. Goldberg DS, MCGee SJ. Pain as a global public health priority. BMC Public Health. 2011;11:770.

2. Gatchel RJ, McGeary DD, McGeary CA, Lippe B. Interdisciplinary chronic pain management: past, present, and future. Am Psychol. 2014;69(2):119-30.

3. Fuchs PN, Peng YB, Boyette-Davis JA, Uhelski ML. The anterior cingulate cortex and pain processing. Front Integr Neurosci. 2014;8:35.

4. LaGraize SC, Borzan J, Peng YB, Fuchs PN. Selective regulation of pain affect following activation of the opioid anterior cingulate cortex system. Exp Neurol. 2006;197(1):22-30.

5. Kata V, Novitch MB, Jones MR, Anyama BO, Helander EM, Kaye AD. Opioid addiction, diversion, and abuse in chronic and cancer pain. Curr Opin Support Palliat Care. 2018;12(2):124-30.

6. Jones JD, Vogelman JS, Luba R, Mumtaz M, Comer SD. Chronic pain and opioid abuse: factors associated with health-related quality of life. Am J Addict. 2017;26(8):815-21.

7. Auvray M, Myin E, Spence $C$. The sensory-discriminative and affectivemotivational aspects of pain. Neurosci Biobehav Rev. 2010;34(2):214-23.

8. Bowers KJ, McAllister KB, Ray M, Heitz C. Ketamine as an adjunct to opioids for acute pain in the emergency department: a randomized controlled trial. Acad Emerg Med. 2017;24(6):676-85.

9. Shikanai H, Hiraide S, Kamiyama H, Kiya T, Oda K, Goto Y, et al. Subanalgesic ketamine enhances morphine-induced antinociceptive activity without cortical dysfunction in rats. J Anesth. 2014;28(3):390-8.

10. Mak P, Broadbear JH, Kolosov A, Goodchild CS. Long-Term antihyperalgesic and opioid-sparing effects of 5-day ketamine and morphine infusion ("Burst Ketamine") in diabetic neuropathic rats. Pain Med. 2015;16(9):1781-93.

11. Chen F, Wang L, Chen S, Li Z, Chen Z, Zhou X, et al. Nasal inhalation of butorphanol in combination with ketamine quickly elevates the mechanical pain threshold in the model of chronic constriction injury to the sciatic nerve of rat. J Surg Res. 2014;186(1):292-6.

12. Jouguelet-Lacoste J, La Colla L, Schilling D, Chelly JE. The use of intravenous infusion or single dose of low-dose ketamine for postoperative analgesia: a review of the current literature. Pain Med. 2015;16(2):383-403

13. Mathews DC, Henter ID, Zarate CA. Targeting the glutamatergic system to treat major depressive disorder: rationale and progress to date. Drugs. 2012;72(10):1313-33.

14. Daly EJ, Singh JB, Fedgchin M, Cooper K, Lim P, Shelton RC, et al. Efficacy and safety of intranasal esketamine adjunctive to oral antidepressant therapy in treatment-resistant depression: a randomized clinical trial. JAMA Psychiatry. 2018;75(2):139-48.

15. Orhurhu V, Orhurhu MS, Bhatia A, Cohen SP. Ketamine infusions for chronic pain: a systematic review and meta-analysis of randomized controlled trials. Anesth Analg. 2019;129(1):241-54.

16. Berman RM, Cappiello A, Anand A, Oren DA, Heninger GR, Charney DS, et al. Antidepressant effects of ketamine in depressed patients. Biol Psychiatry. 2000;47(4):351-4.

17. Zarate CA Jr, Singh JB, Carlson PJ, Brutsche NE, Ameli R, Luckenbaugh DA, et al. A randomized trial of an N-methyl-D-aspartate antagonist in treatment-resistant major depression. Arch Gen Psychiatry. 2006;63(8):856-64.

18. Autry AE, Adachi M, Nosyreva E, Na ES, Los MF, Cheng PF, et al. NMDA receptor blockade at rest triggers rapid behavioural antidepressant responses. Nature. 2011;475(7354):91-5.

19. Petzke F, Clauw DJ, Ambrose K, Khine A, Gracely RH. Increased pain sensitivity in fibromyalgia: effects of stimulus type and mode of presentation. Pain. 2003:105(3):403-13.

20. Scudds RA, Rollman GB, Harth M, McCain GA. Pain perception and personality measures as discriminators in the classification of fibrositis. J Rheumatol. 1987;14(3):563-9.

21. Kehlet $\mathrm{H}$, Jensen TS, Woolf $\mathrm{CJ}$. Persistent postsurgical pain: risk factors and prevention. Lancet. 2006;367(9522):1618-25.

22. Pozek JP, Beausang D, Baratta JL, Viscusi ER. The Acute to chronic pain transition: can chronic pain be prevented? Med Clin North Am. 2016;100(1):17-30.

23. Mansour AR, Farmer MA, Baliki MN, Apkarian AV. Chronic pain: the role of learning and brain plasticity. Restor Neurol Neurosci. 2014:32(1):129-39.
24. Bushnell MC, Ceko M, Low LA. Cognitive and emotional control of pain and its disruption in chronic pain. Nat Rev Neurosci. 2013;14(7):502-11.

25. Bliss TV, Collingridge GL, Kaang BK, Zhuo M. Synaptic plasticity in the anterior cingulate cortex in acute and chronic pain. Nat Rev Neurosci. 2016;17(8):485-96

26. Barthas F, Sellmeijer J, Hugel S, Waltisperger E, Barrot M, Yalcin I. The anterior cingulate cortex is a critical hub for pain-induced depression. Biol Psychiatry. 2015;77(3):236-45.

27. Johansen JP, Fields HL. Glutamatergic activation of anterior cingulate cortex produces an aversive teaching signal. Nat Neurosci. 2004;7(4):398-403.

28. Ma JH, Xiao TH, Chang CW, Gao L, Wang XL, Gao GD, et al. Activation of anterior cingulate cortex produces inhibitory effects on noxious mechanical and electrical stimuli-evoked responses in rat spinal WDR neurons. Eur J Pain. 2011;15(9):895-9.

29. Sikes RW, Vogt BA. Nociceptive neurons in area 24 of rabbit cingulate cortex. J Neurophysiol. 1992;68(5):1720-32.

30. Rothe M, Quilodran R, Sallet J, Procyk E. Coordination of high gamma activity in anterior cingulate and lateral prefrontal cortical areas during adaptation. J Neurosci. 2011:31(31):11110-7.

31. Rainville P, Duncan GH, Price DD, Carrier B, Bushnell MC. Pain affect encoded in human anterior cingulate but not somatosensory cortex. Science. 1997;277(5328):968-71.

32. Davis KD, Taylor SJ, Crawley AP, Wood ML, Mikulis DJ. Functional MRI of pain- and attention-related activations in the human cingulate cortex. J Neurophysiol. 1997;77(6):3370-80.

33. Singh A, Patel D, Li A, Hu L, Zhang Q, Liu Y, et al. Mapping cortical integration of sensory and affective pain pathways. Curr Biol. 2020;30(9):1703-15.

34. Rogers R, Wise RG, Painter DJ, Longe SE, Tracey I. An investigation to dissociate the analgesic and anesthetic properties of ketamine using functional magnetic resonance imaging. Anesthesiology. 2004;100(2):292-301.

35. Niesters M, Khalili-Mahani N, Martini C, Aarts L, van Gerven J, van Buchem MA, et al. Effect of subanesthetic ketamine on intrinsic functional brain connectivity: a placebo-controlled functional magnetic resonance imaging study in healthy male volunteers. Anesthesiology. 2012;117(4):868-77

36. Zhou H, Zhang Q, Martinez E, Dale J, Hu S, Zhang E, et al. Ketamine reduces aversion in rodent pain models by suppressing hyperactivity of the anterior cingulate cortex. Nat Commun. 2018;9(1):3751

37. Guirimand F, Dupont X, Brasseur L, Chauvin M, Bouhassira D. The effects of ketamine on the temporal summation (wind-up) of the $\mathrm{R}(\mathrm{III})$ nociceptive flexion reflex and pain in humans. Anesth Analg. 2000;90(2):408-14.

38. Vyklicky V, Korinek M, Smejkalova T, Balik A, Krausova B, Kaniakova M, et al. Structure, function, and pharmacology of NMDA receptor channels. Physiol Res. 2014;63(Suppl 1):S191-203.

39. Gonzalez J, Jurado-Coronel JC, Avila MF, Sabogal A, Capani F, Barreto GE. NMDARs in neurological diseases: a potential therapeutic target. Int J Neurosci. 2015;125(5):315-27.

40. Inquimbert P, Moll M, Latremoliere A, Tong CK, Whang J, Sheehan GF, et al. NMDA receptor activation underlies the loss of spinal dorsal horn neurons and the transition to persistent pain after peripheral nerve injury. Cell Rep. 2018;23(9):2678-89.

41. Martin D, Lodge D. Ketamine acts as a non-competitive N-methyl-Daspartate antagonist on frog spinal-cord invitro. Neuropharmacology. 1985;24(10):999-1003.

42. Laurido C, Pelissier T, Perez H, Flores F, Hernandez A. Effect of ketamine on spinal cord nociceptive transmission in normal and monoarthritic rats. NeuroReport. 2001;12(8):1551-4.

43. Luo H, Huang Y, Du X, Zhang Y, Green AL, Aziz TZ, et al. Dynamic neural state identification in deep brain local field potentials of neuropathic pain. Front Neurosci. 2018;12:237.

44. Burma NE, Leduc-Pessah H, Fan CY, Trang T. Animal models of chronic pain: advances and challenges for clinical translation. J Neurosci Res. 2017;95(6):1242-56

45. Li N, Lee B, Liu RJ, Banasr M, Dwyer JM, Iwata M, et al. mTOR-dependent synapse formation underlies the rapid antidepressant effects of NMDA antagonists. Science. 2010;329(5994):959-64 
46. Wang J, Goffer Y, Xu D, Tukey DS, Shamir DB, Eberle SE, et al. A single subanesthetic dose of ketamine relieves depression-like behaviors induced by neuropathic pain in rats. Anesthesiology. 2011;115(4):812-21

47. Maeng S, Zarate CA Jr, Du J, Schloesser RJ, McCammon J, Chen G, et al. Cellular mechanisms underlying the antidepressant effects of ketamine: role of alpha-amino-3-hydroxy-5-methylisoxazole-4-propionic acid receptors. Biol Psychiatry. 2008;63(4):349-52.

48. Abdallah CG, Sanacora G, Duman RS, Krystal JH. The neurobiology of depression, ketamine and rapid-acting antidepressants: is it glutamate inhibition or activation? Pharmacol Ther. 2018;190:148-58.

49. Yang Y, Cui Y, Sang K, Dong Y, Ni Z, Ma S, et al. Ketamine blocks bursting in the lateral habenula to rapidly relieve depression. Nature. 2018;554(7692):317-22.

50. Gass N, Becker R, Reinwald J, Cosa-Linan A, Sack M, Weber-Fahr W, et al. Differences between ketamine's short-term and long-term effects on brain circuitry in depression. Transl Psychiat. 2019;9:12.

51. Wang J, Wang J, Xing GG, Li X, Wan Y. Enhanced gamma oscillatory activity in rats with chronic inflammatory pain. Front Neurosci. 2016;10:489.

52. May ES, Nickel MM, Ta Dinh S, Tiemann L, Heitmann H, Voth I, et al. Prefrontal gamma oscillations reflect ongoing pain intensity in chronic back pain patients. Hum Brain Mapp. 2019;40(1):293-305.

53. Tiemann L, Schulz E, Gross J, Ploner M. Gamma oscillations as a neuronal correlate of the attentional effects of pain. Pain. 2010;150(2):302-8.

54. Fu B, Wen SN, Wang B, Wang K, Zhang JY, Liu SJ. Acute and chronic pain affects local field potential of the medial prefrontal cortex in different band neural oscillations. Mol Pain. 2018;14:1744806918785686.

55. Li X, Zhao Z, Ma J, Cui S, Yi M, Guo H, et al. Extracting Neural oscillation signatures of laser-induced nociception in pain-related regions in rats. Front Neural Circuits. 2017;11:71.

56. Veerasarn $P$, Stohler CS. The effect of experimental muscle pain on the background electrical brain activity. Pain. 1992;49(3):349-60.

57. Zhang Q, Xiao Z, Huang C, Hu S, Kulkarni P, Martinez E, et al. Local field potential decoding of the onset and intensity of acute pain in rats. Sci Rep. 2018;8(1):8299.

58. Wu JH, Chang WD, Hsieh CW, Jiang JA, Fang W, Shan YC, et al. Effect of low-level laser stimulation on EEG. Evid Based Complement Alternat Med. 2012;2012:951272

59. Dubin AE, Patapoutian A. Nociceptors: the sensors of the pain pathway. J Clin Invest. 2010:120(11):3760-72.

60. Lewin GR, Moshourab R. Mechanosensation and pain. J Neurobiol. 2004;61(1):30-44.

61. Gold MS, Gebhart GF. Nociceptor sensitization in pain pathogenesis. Nat Med. 2010;16(11):1248-57.

62. Cavanaugh DJ, Lee H, Lo L, Shields SD, Zylka MJ, Basbaum Al, et al. Distinct subsets of unmyelinated primary sensory fibers mediate behavioral responses to noxious thermal and mechanical stimuli. Proc Natl Acad Sci USA. 2009;106(22):9075-80.

63. Viana F. Nociceptors: thermal allodynia and thermal pain. Handb Clin Neurol. 2018;156:103-19.

64. Julius D. TRP channels and pain. Annu Rev Cell Dev Biol. 2013;29:355-84

65. Apkarian AV, Bushnell MC, Treede RD, Zubieta JK. Human brain mechanisms of pain perception and regulation in health and disease. Eur $J$ Pain. 2005;9(4):463-84.

66. Gungor NZ, Johansen J. A Chronic Pain in the ACC. Neuron. 2019;102(5):903-5.

67. Li XY, Ko HG, Chen T, Descalzi G, Koga K, Wang H, et al. Alleviating neuropathic pain hypersensitivity by inhibiting PKMzeta in the anterior cingulate cortex. Science. 2010;330(6009):1400-4.

68. Qu C, King T, Okun A, Lai J, Fields HL, Porreca F. Lesion of the rostral anterior cingulate cortex eliminates the aversiveness of spontaneous neuropathic pain following partial or complete axotomy. Pain. 2011:152(7):1641-8

69. Tan LL, Oswald MJ, Heinl C, Retana Romero OA, Kaushalya SK, Monyer $\mathrm{H}$, et al. Gamma oscillations in somatosensory cortex recruit prefrontal and descending serotonergic pathways in aversion and nociception. Nat Commun. 2019;10(1):983.
70. Zhang Q, Manders T, Tong AP, Yang R, Garg A, Martinez E, et al Chronic pain induces generalized enhancement of aversion. Elife. 2017:6:e25302.

71. Hutchison WD, Davis KD, Lozano AM, Tasker RR, Dostrovsky JO. Pain-related neurons in the human cingulate cortex. Nat Neurosci. 1999;2(5):403-5.

72. Kudel I, Edwards RR, Kozachik S, Block BM, Agarwal S, Heinberg LJ, et al. Predictors and consequences of multiple persistent postmastectomy pains. J Pain Symptom Manag. 2007;34(6):619-27.

73. Scott CEH, Howie CR, MacDonald D, Biant LC. Predicting dissatisfaction following total knee replacement a prospective study of 1217 patients. J Bone Joint Surg Br. 2010;92(9):1253-8.

74. Buzsaki G, Leung LWS, Vanderwolf CH. Cellular bases of hippocampal eeg in the behaving rat. Brain Res Rev. 1983;6(2):139-71.

75. Buzsaki G, Wang XJ. Mechanisms of gamma oscillations. Annu Rev Neurosci. 2012:35:203-25

76. Garcia LS, Comim CM, Valvassori SS, Reus GZ, Barbosa LM, Andreazza $A C$, et al. Acute administration of ketamine induces antidepressantlike effects in the forced swimming test and increases BDNF levels in the rat hippocampus. Prog Neuropsychopharmacol Biol Psychiatry. 2008;32(1):140-4

77. Zhou W, Wang N, Yang C, Li XM, Zhou ZQ, Yang JJ. Ketamine-induced antidepressant effects are associated with AMPA receptors-mediated upregulation of mTOR and BDNF in rat hippocampus and prefrontal cortex. Eur Psychiatry. 2014;29(7):419-23.

78. Yang C, Hu YM, Zhou ZQ, Zhang GF, Yang JJ. Acute administration of ketamine in rats increases hippocampal BDNF and mTOR levels during forced swimming test. Ups J Med Sci. 2013;118(1):3-8.

79. Fukumoto K, Toki H, lijima M, Hashihayata T, Yamaguchi Jl, Hashimoto $\mathrm{K}$, et al. Antidepressant potential of (R)-ketamine in rodent models: comparison with (S)-ketamine. J Pharmacol Exp Ther. 2017:361(1):9-16.

80. Fukumoto K, lijima M, Chaki S. The antidepressant effects of an mGlu2/3 receptor antagonist and ketamine require AMPA receptor stimulation in the MPFC and subsequent activation of the 5-HT neurons in the DRN. Neuropsychopharmacology. 2016;41(4):1046-56.

81. Koike H, lijima M, Chaki S. Involvement of AMPA receptor in both the rapid and sustained antidepressant-like effects of ketamine in animal models of depression. Behav Brain Res. 2011;224(1):107-11.

82. Llamosas N, Perez-Caballero L, Berrocoso E, Bruzos-Cidon C, Ugedo L, Torrecilla M. Ketamine promotes rapid and transient activation of AMPA receptor-mediated synaptic transmission in the dorsal raphe nucleus. Prog Neuropsychopharmacol Biol Psychiatry. 2019;88:243-52.

83. Shen M, LV D, Liu X, Li S, Chen Y, Zhang Y, et al. Essential roles of neuropeptide VGF regulated TrkB/mTOR/BICC1 signaling and phosphorylation of AMPA receptor subunit GluA1 in the rapid antidepressant-like actions of ketamine in mice. Brain Res Bull. 2018;143:58-65.

84. Zhang K, Yamaki VN, Wei Z, Zheng Y, Cai X. Differential regulation of GluA1 expression by ketamine and memantine. Behav Brain Res. 2017:316:152-9.

85. Akeju O, Song AH, Hamilos AE, Pavone KJ, Flores FJ, Brown EN, et al. Electroencephalogram signatures of ketamine anesthesia-induced unconsciousness. Clin Neurophysiol. 2016;127(6):2414-22.

86. Amat-Foraster M, Jensen AA, Plath N, Herrik KF, Celada P, Artigas F. Temporally dissociable effects of ketamine on neuronal discharge and gamma oscillations in rat thalamo-cortical networks. Neuropharmacology. 2018;137:13-23.

87. Ma L, Skoblenick K, Johnston K, Everling S. Ketamine alters lateral prefrontal oscillations in a rule-based working memory task. J Neurosci. 2018;38(10):2482-94

88. Vlisides PE, Bel-Bahar T, Nelson A, Chilton K, Smith E, Janke E, et al. Subanaesthetic ketamine and altered states of consciousness in humans. $\mathrm{Br}$ J Anaesth. 2018;121(1):249-59.

89. Li M, Woelfer M, Colic L, Safron A, Chang CT, Heinze HJ, et al. Default mode network connectivity change corresponds to ketamine's delayed glutamatergic effects. Eur Arch Psy Clin N. 2020;270(2):207-16.

90. Carlen M, Meletis K, Siegle JH, Cardin JA, Futai K, Vierling-Claassen $D$, et al. A critical role for NMDA receptors in parvalbumin interneurons for gamma rhythm induction and behavior. Mol Psychiatr. 2012;17(5):537-48.

91. Boeijinga P, Danjou P, Patroneva A, Smith MA, Quirk M. Low-trapping NMDA channel blocker AZD6765 increases gamma-band EEG without 
dissociative side-effects: a comparison with ketamine in healthy volunteers. Int J Neuropsychoph. 2012;15:142.

92. Quirk M, Ploeger B, Borg N, Piser T, Doherty J. Effects of low-trapping NMDA channel blocker AZD6765 on gamma-band EEG and psychotomimetic liability: a comparison to ketamine in freely behaving rats. Int $J$ Neuropsychoph. 2012;15:153.

93. Anderson PM, Pinault D, O'Brien TJ, Jones NC. Chronic administration of antipsychotics attenuates ongoing and ketamine-induced increases in cortical gamma oscillations. Int J Neuropsychoph. 2014;17(11):1895-904.

94. Furth KE, McCoy AJ, Dodge C, Walters JR, Buonanno A, Delaville C. Neuronal correlates of ketamine and walking induced gamma oscillations in the medial prefrontal cortex and mediodorsal thalamus. PLOS ONE. 2017;12(11):e0186732

95. Ye T, Bartlett MJ, Schmit MB, Sherman SJ, Falk T, Cowen SL. Ten-hour exposure to low-dose ketamine enhances corticostriata cross-frequency coupling and hippocampal broad-band gamma oscillations. Front Neural Circuit. 2018;12:61.

96. Duman RS, Aghajanian GK. Synaptic dysfunction in depression: potential therapeutic targets. Science. 2012;338(6103):68-72.

97. Hua T, Chen B, Lu D, Sakurai K, Zhao S, Han BX, et al. General anesthetics activate a potent central pain-suppression circuit in the amygdala. Nat Neurosci. 2020;23:854-68.

98. Buzsaki G, Anastassiou CA, Koch C. The origin of extracellular fields and currents-EEG, ECoG. LFP and spikes Nat Rev Neurosci. 2012;13(6):407-20.
99. Zhang ZG, Hu L, Hung YS, Mouraux A, lannetti GD. Gamma-band oscillations in the primary somatosensory cortex - a direct and obligatory correlate of subjective pain intensity. J Neurosci. 2012;32(22):7429-38.

100. Gross J, Schnitzler A, Timmermann L, Ploner M. Gamma oscillations in human primary somatosensory cortex reflect pain perception. PLoS Biol. 2007;5(5):e133.

101. Schulz E, Zherdin A, Tiemann L, Plant C, Ploner M. Decoding an individual's sensitivity to pain from the multivariate analysis of EEG data. Cereb Cortex. 2012;22(5):1118-23.

102. Chen Z, Zhang Q, Tong APS, Manders TR, Wang J. Deciphering neuronal population codes for acute thermal pain. J Neural Eng. 2017;14(3):036023.

103. Lee M, Manders TR, Eberle SE, Su C, D'Amour J, Yang R, et al. Activation of corticostriatal circuitry relieves chronic neuropathic pain. J Neurosci. 2015;35(13):5247-59.

104. Thomson DJ. Spectrum estimation and harmonic-analysis. Proc IEEE. 1982;70(9):1055-96.

105. Bokil H, Andrews P, Kulkarni JE, Mehta S, Mitra PP. Chronux: a platform for analyzing neural signals. J Neurosci Methods. 2010;192(1):146-51.

\section{Publisher's Note}

Springer Nature remains neutral with regard to jurisdictional claims in published maps and institutional affiliations.
Ready to submit your research? Choose BMC and benefit from:

- fast, convenient online submission

- thorough peer review by experienced researchers in your field

- rapid publication on acceptance

- support for research data, including large and complex data types

- gold Open Access which fosters wider collaboration and increased citations

- maximum visibility for your research: over $100 \mathrm{M}$ website views per year

At BMC, research is always in progress.

Learn more biomedcentral.com/submissions 